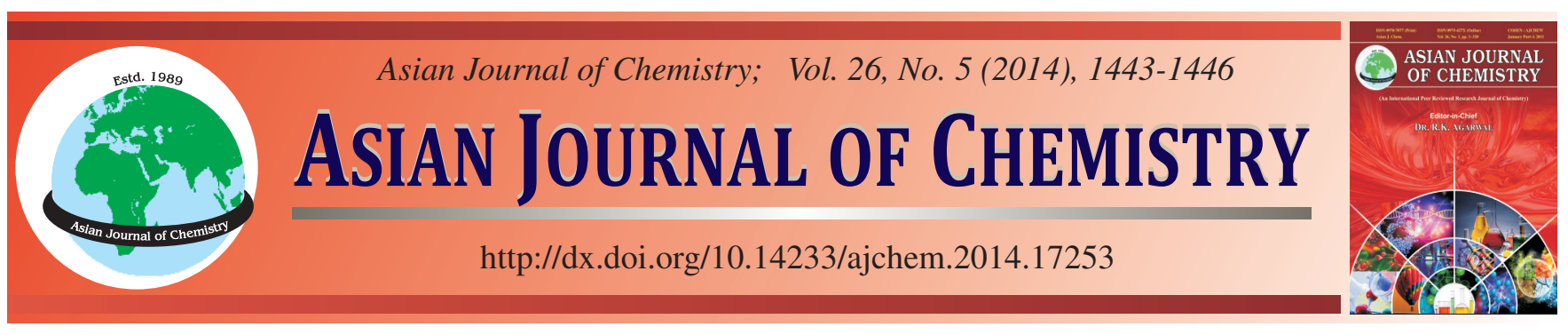

\title{
Study on Property and Mechanism of Adsorption of Chlorogenic Acids by Zinc-Containing D401 Macroporous Resin $\dagger$
}

\author{
Han Xuan ${ }^{*}$, Fazhi Xie, Ying Wang, Tian CaO, Yan Chen and Binbin Xia
}

School of Materials Science and Chemical Engineering, Anhui Jianzhu University, Hefei 230022, P.R. China

*Corresponding author: E-mail: xuanhan@ahjzu.edu.cn

\begin{abstract}
The property and mechanism of the adsorption of chlorogenic acids by zinc-containing D401 macroporous adsorption resin was analyzed. The isothermal adsorption property, the kinetic characteristics, the dynamic adsorption and desorption characteristics of the adsorption of chlorogenic acids by zinc-containing D401 macroporous resin were investigated by the adsorption test. The results indicate that the equilibrium time of adsorption is $2 \mathrm{~h}$. The adsorption follow the second-order kinetic equation with the adsorption rate constant $0.0024 \mathrm{~g}$ $\mathrm{mg}^{-1} \mathrm{~h}^{-1}$; the adsorption isotherm data correspond well to the Langmuir equation with the maximum adsorption capacity $\left(\mathrm{Q}_{\mathrm{m}}\right) 43.10 \mathrm{mg} \mathrm{g}^{-1}$ and the adsorption is a physical adsorption. The adsorption flow rate is $2 \mathrm{BV} / \mathrm{h}$; the solution concentration is $1.3 \mathrm{mg} / \mathrm{mL}$ and $\mathrm{pH}$ value is 1. The adsorbed chlorogenic acids can be eluted from zinc-containing D401 macroporous resin by $70 \%$ ethanol.
\end{abstract}

Keywords: Zinc-containing D401 macroporous resin, Adsorption, Chlorogenic acid.

\section{INTRODUCTION}

The chlorogenic acid is an essential physiological active substance with antibacterial, antiviral, antitumor, hepatic protective and choleretic effects. It helps raising the leukocytes, lowering blood pressure and blood lipid and scavenging free radicals. It has found wide applications in food, pharmaceutical and chemical industries ${ }^{1}$. With the further studies on the efficacies of chlorogenic acid and its analogues, the values of chlorogenic acid from natural plants have been appreciated more than before. Therefore, the search for effective methods for separation and purification of chlorogenic acids has important significance on developing effective constituents in natural products.

Macroporous adsorption resin is a class of organic polymers adsorbent developed over the past decade ${ }^{2}$. It has physical and chemical stabilities. The adsorption selectivity is unique and not affected by inorganic existence. It is also featured by easy regeneration, mild desorption conditions, long life cycle and costs saving. Macroporous adsorption resin has been widely used in the separation of water-soluble plant active ingredients. In this paper, the D401 resin was structurally modified with zinc ions and the chlorogenic acid adsorption properties and mechanism were analyzed so as to provide a reference for the chlorogenic acid separation and purification.

\section{EXPERIMENTAL}

D401 macroporous adsorption resin (Chemical Plant of Nankai University); control products of chlorogenic acid for content determination (ID 110715-200912, the China drugs and Biological Products Inspection Institute); methanol; acetonitrile of chromatographic purity; glacial acetic acid; hydrochloric acid; sodium hydroxide; zinc sulfate; ferric chloride of analytical purity; $95 \%$ ethanol (China Pharmaceutical Group Chemical Reagents Company, Shanghai).

$\mathrm{ZnSO}_{4}$ solution $(10 \%)$ and $\mathrm{FeCl}_{3}$ solution $(10 \%)$ was mixed with pre-treated D401 macroporous resin ${ }^{3}$ with the ratio of 10:1, respectively, the reaction was performed at room temperature for $48 \mathrm{~h}$. The product was washed with deionized water until metal ions could not be detected and then dried in $60{ }^{\circ} \mathrm{C}$.

TU-1800PC UV-VIS spectrophotometer (Being Puxi General Instrument Co., Ltd); Sartorius BS22S Electronic balance (German Sartorius); KQ5200B Ultrasonic cleaning instrument (Kun Shan Ultrasonic Instruments Co., Ltd); Magnetic stirring electric heating jacket (Jintan Ronghua Instrunent Manufacture Co. Ltd.); Agilent1200 Liquid chromatograph (Agilent Technologies).

Preparation of standard solutions of chlorogenic acid: $3.7 \mathrm{mg}$ of control product of chlorogenic acid was weighted

†Presented at The 7th International Conference on Multi-functional Materials and Applications, held on 22-24 November 2013, Anhui University of Science \& Technology, Huainan, Anhui Province, P.R. China 
precisely and put in $10 \mathrm{~mL}$ volumetric flask. The methanol was added into the flask to dilute to the scale and mixed with the control product well.

HPLC analysis of chlorogenic acid: The HPLC method was used to determine samples with a Hypersil BDS $\mathrm{C}_{18}$ column $(250 \mathrm{~mm} \times 4.6 \mathrm{~mm}, 5 \mu \mathrm{m})$. The column oven temperature was fixed at $30^{\circ} \mathrm{C}$. A mobile phase consisting of methanol and 0.6 $\%$ glacial acetic acid was applied for the elution: $15 / 85$ with the flow rate of $0.7 \mathrm{~mL} / \mathrm{min}$. Diode array detection was used to detect chlorogenic acid at $327 \mathrm{~nm}$. The results indicated that the working calibration curve based on standard solutions of chlorogenic acid showed excellent linearity over the range of $0.37-1.85 \mu \mathrm{g}$. The regression line for chlorogenic acid was as following eqn. 1 :

$$
\mathrm{A}=4289.75 \mathrm{C}-56.44(\mathrm{r}=0.9997, \mathrm{n}=5)
$$

where A represented the peak area of chlorogenic acid and $\mathrm{C}$ was the chlorogenic acid concentrations $(\mu \mathrm{g} / \mathrm{mL})$.

Static adsorption tests: The adsorption data of chlorogenic acid on some resins were determined as follows: $0.1 \mathrm{~g}$ (dry weight) of pretreated resins, added by $25 \mathrm{~mL}$ chlorogenic acid solution of $1.26 \mathrm{mg} / \mathrm{mL}$ concentration, was put in a flask and stirred with a magnetic stirrer. The concentration of chlorogenic acid was measured at regular intervals. The preliminary choice of the resins was evaluated by the adsorption capacity. The adsorption capacity of the resin at equilibrium was calculated and the adsorption dynamic curve was plotted accordingly. The following equation was used to quantify the capacity of adsorption.

$$
\mathrm{q}_{\mathrm{e}}=\frac{\left(\mathrm{C}_{0}-\mathrm{C}_{\mathrm{e}}\right) \mathrm{V}}{\mathrm{W}}
$$

where $\mathrm{q}_{\mathrm{e}}$ is the adsorption capacity at adsorption equilibrium (mg/g resin), $\mathrm{C}_{0}$ and $\mathrm{C}_{\mathrm{e}}$ are the initial and equilibrium concentrations of chlorogenic acid in the solutions, respectively ( $\mathrm{mg}$ / $\mathrm{mL}$ ) and $\mathrm{W}$ is the weight of the dry resin $(\mathrm{g})$.

Adsorption kinetics and isotherms: The adsorption kinetics of the macroporous resin was studied by contacting $50 \mathrm{~mL}$ of chlorogenic acid solution of $0.2 \mathrm{mg} / \mathrm{mL}$ concentration with $100 \mathrm{mg}$ zinc-containing D401 resin on a flask and stirred with a magnetic stirrer. The concentration of chlorogenic acid in the adsorption solution was determined by HPLC at different time until equilibration. In order to investigate effect of temperature and initial concentration on chlorogenic acid adsorption, the adsorption isotherms on zinc-containing D401 resin were performed by adding $100 \mathrm{mg}$ zinc-containing D401 resin and $20 \mathrm{~mL}$ of sample solution of chlorogenic acid into each flask at different initial concentrations and temperatures.

Langmuir and Freundlich models are used to compare adsorption of resins ${ }^{4}$. The Langmuir equation can be used to describe a monolayer adsorption, whereas the Freundlich equation can be used to describe a monolayer adsorption as well as a multilayer adsorption.

$$
\text { Langmuir equation: } \mathrm{q}_{\mathrm{e}}=\frac{\mathrm{q}_{\max } \mathrm{a}_{\mathrm{L}} \mathrm{C}_{\mathrm{e}}}{1+\mathrm{a}_{\mathrm{L}} \mathrm{C}_{\mathrm{e}}}
$$

$$
\text { Freundlich equation: } \mathrm{q}_{\mathrm{e}}=\mathrm{K}_{\mathrm{F}} \mathrm{C}_{\mathrm{e}}^{1 / \mathrm{n}}
$$

where $\mathrm{q}_{\mathrm{e}}$ and $\mathrm{C}_{\mathrm{e}}$ represent the same parameters as in formulae (2), $\mathrm{q}_{\max }$ the theoretically calculated maximum adsorption capacity ( $\mathrm{mg} / \mathrm{g}$ resin), $\mathrm{a}_{\mathrm{L}}$ is the adsorption equilibrium constant related to the affinity between the adsorbent and adsorbate, $\mathrm{K}_{\mathrm{F}}$ the Freundlich constant, an indicator of adsorption capacity and $1 / \mathrm{n}$ an empirical constant related to the magnitude of the adsorption driving force.

Dynamic adsorption and desorption tests: Chromatography column was packed with $2.6 \mathrm{~g}$ zinc-containing D401 resin. The column volume was $5 \mathrm{~mL}$. The chlorogenic acid solution was applied onto the column and passed through the resin with a certain velocity. The eluate was collected in different tubes and the concentrations of the chlorogenic acid were measured. Factors influencing the chlorogenic acid adsorption such as initial concentration of the chlorogenic acid solutions (0.2-1.7 mg/mL), $\mathrm{pH}$ (1-5), the velocities (1-4 BV/h) of solution passed through the resin were studied.

The chlorogenic acid solution of a certain concentration was passed through the resin, after the adsorption reached equilibrium, the column was eluted stepwise with deionized water of $4 \mathrm{BV}$, ethanol of 50, 60, 70, 80 and $95 \%$ concentrations of $2 \mathrm{BV} / \mathrm{h}$ velocity. The eluate was collected sequentially in different tubes. The concentrations of the chlorogenic acid were measured for each fraction.

\section{RESULTS AND DISCUSSION}

Preliminary choice of the resins: The adsorption amounts of chlorogenic acid by different resins are shown in Table-1. The data suggested that the zinc-containing resin has the best adsorption performance among the three. Therefore the following experiments were carried out with the zinc-containing resin.

\begin{tabular}{cccc}
\multicolumn{4}{c}{ TABLE-1 } \\
RESULT OF RESIN SCREENING \\
\hline $\begin{array}{c}\text { Zn-containing } \\
\text { resin }\end{array}$ & $\begin{array}{c}\text { Fe-containing } \\
\text { resin }\end{array}$ & $\begin{array}{c}\text { Unmodified } \\
\text { resin }\end{array}$ \\
\hline $\begin{array}{c}\text { Amount of } \\
\text { adsorption }(\mathrm{mg} / \mathrm{g})\end{array}$ & 30.65 & 24.55 & 24.20 \\
\hline
\end{tabular}

Kinetics of adsorption of chlorogenic acid by zinccontaining D401 resin: The adsorption of chlorogenic acid by zinc-containing D401 resin can be divided into the initial rapid adsorption stage (the first $2 \mathrm{~h}$ ) and the subsequent slow adsorption stage. The adsorption reached equilibrium after $2 \mathrm{~h}$ (Fig. 1). The adsorption data were applied to the pseudo second-order model curve fitting 5 .

$$
\frac{\mathrm{t}}{\mathrm{q}_{\mathrm{t}}}=\frac{1}{\mathrm{k}_{2} \mathrm{q}_{\mathrm{e}}^{2}}+\frac{1}{\mathrm{q}_{\mathrm{e}}} \mathrm{t}
$$

where $\mathrm{q}_{\mathrm{e}}$ and $\mathrm{q}_{\mathrm{t}}$ were pseudo adsorption capacity at equilibrium and time $\mathrm{t}\left(\mathrm{mg} / \mathrm{g}\right.$ dry resin), respectively. $\mathrm{k}_{2}$ was the rate constant of pseudo-second-order adsorption.

The results suggested that the adsorption of chlorogenic acid by zinc-containing D401 resin followed the second-order kinetic equation with a good linear relationship. The variance $\left(\mathrm{r}^{2}\right)$ is 0.9962 . The constant of equilibrium rate $\left(\mathrm{k}_{2}\right)$ is 0.0024 $\mathrm{g} \mathrm{mg}^{-1} \mathrm{~h}^{-1}$. The adsorption amount at equilibrium $\left(\mathrm{q}_{\mathrm{e}}\right)$ is 43.10 $\mathrm{mg} \mathrm{g}^{-1}$. 


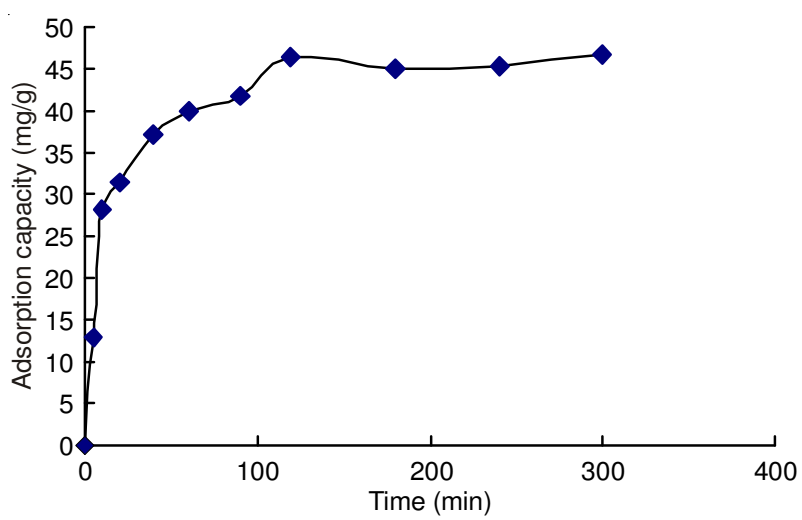

Fig. 1. Kinetics of the adsorption of chlorogenic acid by zinc-containing D401 resin

Isotherm of adsorption of chlorogenic acid by zinccontaining D401 resin: The isotherm of the adsorption of chlorogenic acid is shown in Fig. 2. The result in Table-2 suggested that Langmuir adsorption isotherm equation has a better ability than Freundlich equation to describe zinc-containing D401 resin's adsorption of chlorogenic acid. The reasons are probably that Freundlich equation assumes that the adsorption of molecules on medium is multilayer adsorption and that the surface on the solid adsorbent is even, i.e., the energies of different adsorption spots on the surface are the same and the possibility of monolayer adsorption in the solution is higher ${ }^{6}$.

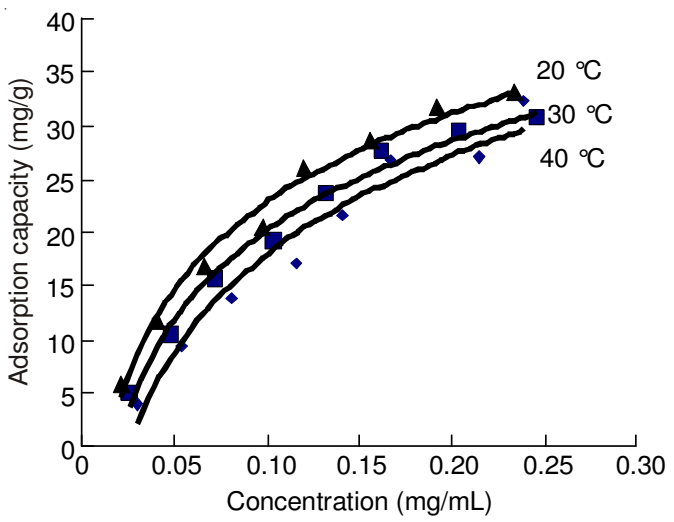

Fig. 2. Isotherm of adsorption of chlorogenic acid by zinc-containing D401 resin

\begin{tabular}{cccc|ccc}
\multicolumn{7}{c}{ TABLE-2 } \\
\multicolumn{7}{c}{$\begin{array}{c}\text { CORRELATED PARAMETERS FOR ADSORPTION } \\
\text { ISOTHERMS OF CHLOROGENIC ACID }\end{array}$} \\
\hline \multirow{2}{*}{$\mathrm{T}\left({ }^{\circ} \mathrm{C}\right)$} & \multicolumn{2}{c}{ Langmuir equation } & \multicolumn{3}{c}{ Freundlich equation } \\
\cline { 2 - 7 } & $\mathrm{q}_{\mathrm{m}}$ & $\mathrm{K}_{\mathrm{L}}$ & $\mathrm{R}^{2}$ & $\mathrm{~K}_{\mathrm{f}}$ & $\mathrm{n}$ & $\mathrm{R}^{2}$ \\
\hline 20 & 70.92 & 294.12 & 0.9964 & 102.80 & 1.40 & 0.9768 \\
30 & 113.64 & 212.77 & 0.9947 & 105.80 & 1.26 & 0.9736 \\
40 & 120.48 & 175.44 & 0.9913 & 130.35 & 1.04 & 0.9774 \\
\hline
\end{tabular}

Leaking curve of zinc-containing D401 resin: The dynamic adsorption curve is shown in Fig. 3. The data indicate that more than $5 \%$ leakage happened when the sample applied to the column reached $7 \mathrm{BV}$, the effluent chlorogenic acid content increased siginificantly when sample reached $9 \mathrm{BV}$, leaking more than $10 \%$. The results suggest that the zinc-containing D401 resin could adsorb $8 \mathrm{BV}$ sample solution and $5 \mathrm{~mL}$ resin adsorbed $34 \mathrm{mg}$ chlorogenic acid in total.

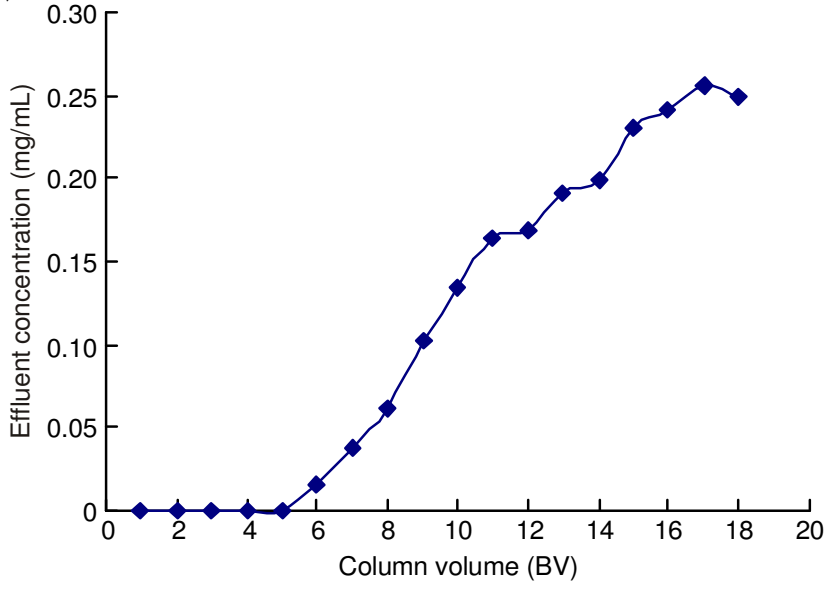

Fig. 3. Isotherm of adsorption of chlorogenic acid by zinc-containing resin

Effect of initial concentrations on the adsorption amount: The effect of initial concentrations on the adsorption amount is shown in Fig. 4. As can be seen, the optimum of the sample's original concentration is $1.3 \mathrm{mg} / \mathrm{mL}$.

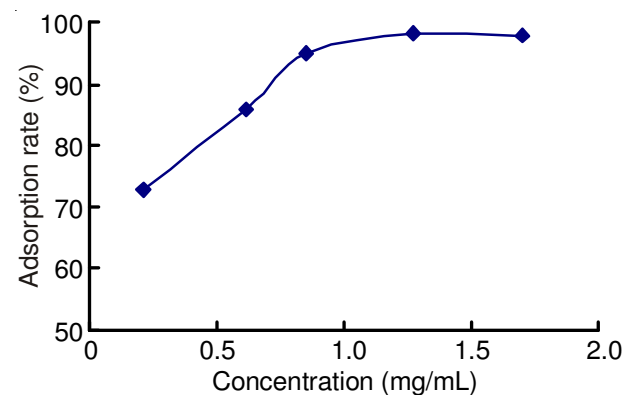

Fig. 4. Effect of sample solution's concentrations on the adsorption amount

Effect of adsorption flow rate on the adsorption amount: The effect of the flow rate on the adsorption amount is shown in Table-3. As can be seen, with the increase of the adsorption flow rate, the adsorption rate of chlorogenic acid decreased. However, if the flow rate is too slow, the adsorption time will increase and the production cycle will be prolonged along with the increase of cost. The adsorption rates are generally high when the flow rate falls between 1 to $2 \mathrm{BV} / \mathrm{h}$. Taking other factors into consideration, $2 \mathrm{BV} / \mathrm{h}$ is chosen as the optimal adsorption flow rate.

\begin{tabular}{cc} 
TABLE-3 \\
EFFECT OF DIFFERENT ADSORPTION FLOW \\
RATES ON THE ADSORPTION PERFORMACE \\
\hline Flow rate $(\mathrm{BV} / \mathrm{h})$ & Adsorption rate $(\%)$ \\
\hline 1 & 91.50 \\
2 & 85.89 \\
3 & 73.84 \\
4 & 60.32 \\
\hline
\end{tabular}

Effect of sample solution's pH on the adsorption amount: The effect of solution's $\mathrm{pH}$ on the adsorption performance is shown in Fig. 5. As can be seen, the solution's pH has a great effect on the adsorption of chlorogenic acid. The maximum adsorption can be achieved when the adsorption solution's $\mathrm{pH}$ is 1. Chlorogenic acid tends to be dissociated into ionic state under alkaline condition. 


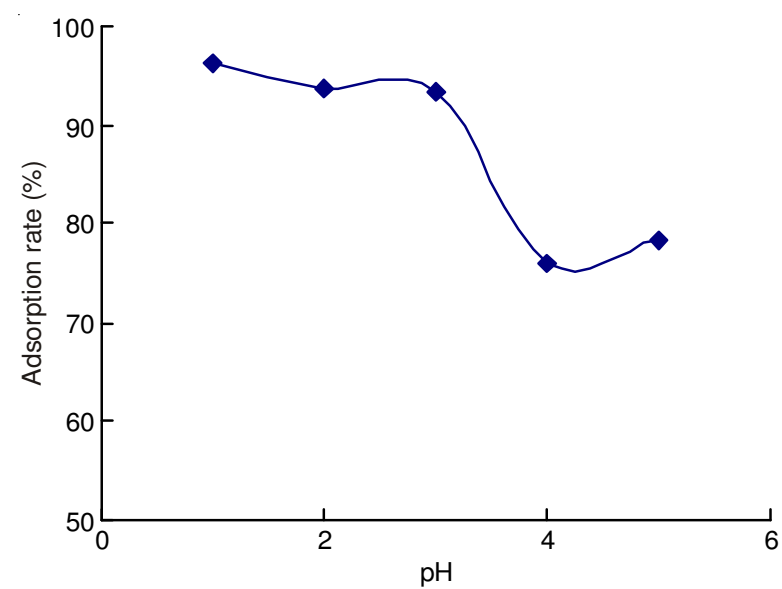

Fig. 5. Effect of sample solution's pH on the adsorption amount

Selection of the eluent and the desorption technology: The effect of ethanol of different concentrations on eluting the adsorption saturated resin is shown in Fig. 6. Data from number 1 to 4 on the $\mathrm{X}$ axis, the eluent are water, 5 to 8 are $50 \%$ ethanol, 9 to 12 are $60 \%$ ethanol, 13 to 16 are $70 \%$ ethanol, 17 to 20 are $80 \%$ ethanol, 21 to 24 are $95 \%$ ethanol. The figure suggests that within the range of 50 to $70 \%$ ethanol, the desorption efficiency increased with the increase of ethanol concentration. When the ethanol concentration is $80 \%$, the eluent concentration is very low. Consider the cost of the eluent, $70 \%$ ethanol will be used as the eluent.

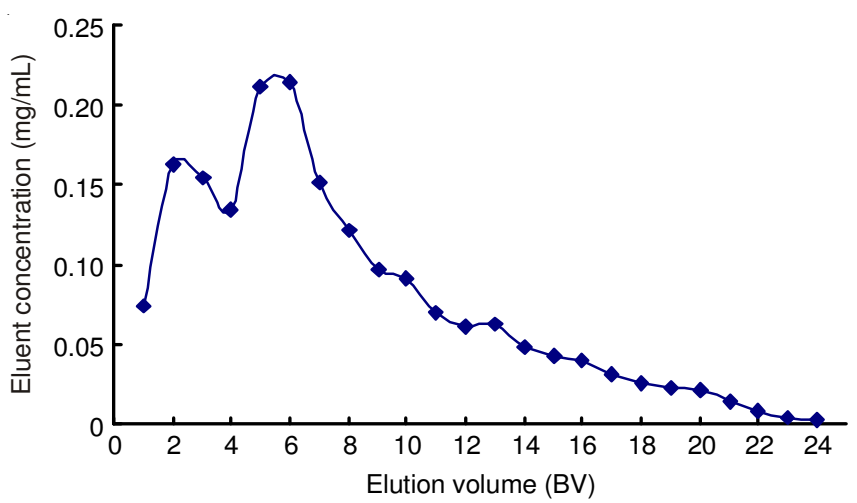

Fig. 6. Dynamic elution curve

Preliminary study on the mechanism of adsorption: Sticking probability (S) can reflect the mechanism of the interaction between the adsorbate and the adsorbent. It depends on the coverage of the adsorbate on the adsorbent $(\theta)$, the activation energy and temperatures. The relationship between sticking probability and the parameters can be expressed in the following equation ${ }^{7}$ :

$$
\begin{gathered}
\ln (1-\theta)=\ln S+\frac{E_{a}}{R T} \\
\theta=1-\frac{C_{e}}{C_{0}}
\end{gathered}
$$

In eqn. $7, \mathrm{C}_{0}$ and $\mathrm{C}_{\mathrm{e}}$ are the concentrations of chlorogenic acid in the begining and at equilibrium $(\mathrm{mg} / \mathrm{L})$. When sticking probability is greater than 1 , there is no adsorption. When sticking probability is equal to 1 , the physical adsorption equals to the chemical adsorption. When sticking probability value is greater than 0 but smaller than 1 , the physical adsorption dominates. When sticking probability equals to 0 , the chemical adsorption dominates.

According to the eqn. 6, the sticking probability sticking probability is 0.0017 and the activation energy $\mathrm{E}_{\mathrm{a}}$ is 0.2063 $\mathrm{kJ} / \mathrm{mol}$. The sticking probability value is less than 1 , suggesting that the adsorption is a physical adsorption. The activation energy is relatively small, further indicating that it is the physical adsorption that dominates.

\section{Conclusion}

The optimal adsorption parameters are the follwoing: the dynamic adsorption flow rate is $2 \mathrm{BV} / \mathrm{h}$; the solution's concentration is $1.3 \mathrm{mg} / \mathrm{mL}$ and the $\mathrm{pH}$ is 1 . The equilibrium time for adsorption is $2 \mathrm{~h}$. The adsorption followed the second-order kinetic equation with the adsorption rate constant is $0.0024 \mathrm{~g}$ $\mathrm{mg}^{-1} \mathrm{~h}^{-1}$. The adsorption isotherm data are well represented by the Langmuir isotherm model with the maximum adsorption capacity $\left(\mathrm{Q}_{\mathrm{m}}\right)$ of $43.10 \mathrm{mg} \mathrm{g}^{-1}$. The sticking probability and activation energy are 0.0017 and $0.2063 \mathrm{~kJ} / \mathrm{mol}$. The adsorption is a physical adsorption. The adsorbed chlorogenic acids can be separated from zinc-containing D401 macroporous adsorption resin by eluting with $70 \%$ ethanol.

\section{ACKNOWLEDGEMENTS}

This work was supported by the National Natural Science Foundation of China (21107001).

\section{REFERENCES}

1. A. Zhang and Q. Ma, Chin. Tradit. Herbal Drugs, 32, 173 (2001).

2. J. Zhou, Y. Ding and J. Chin, Hosp. Pharm., 36, 69 (2006).

3. D.S. Grzegorczyk and G. Carta, Chem. Eng. Sci., 51, 807 (1996).

4. A. Di Mauro, E. Arena, B. Fallico, A. Passerini and E. Maccarone, J. Agric. Food Chem., 50, 5968 (2002).

5. A. Gücek, S. Sener, S. Bilgen and M.A. Mazmanci, J. Colloid Interf. Sci., 286, 53 (2005).

6. P. Atkins and J. Paula, Atkin's Physical Chemistry, Oxford University Press, UK, edn 7, p. 989 (2005).

7. M. Horsfall Jr. and A.I. Spiff, J. Sci. Ind. Res. (India), 64, 613 (2005). 